\title{
Development and In Vitro Evaluation of Liposomes Using Soy Lecithin to Encapsulate Paclitaxel
}

\author{
Thi Lan Nguyen, ${ }^{1,2}$ Thi Hiep Nguyen, ${ }^{3}$ and Dai Hai Nguyen ${ }^{1}$ \\ ${ }^{1}$ Institute of Applied Materials Science, Vietnam Academy of Science and Technology, 01 TL29, District 12, Ho Chi Minh City, Vietnam \\ ${ }^{2}$ Can Tho University, 3/2 Street, Ninh Kieu District, Can Tho City, Vietnam \\ ${ }^{3}$ Tissue Engineering and Regenerative Medicine Group, Department of Biomedical Engineering, International University, \\ Vietnam National University-HCMC (VNU-HCMC), Ho Chi Minh City 70000, Vietnam
}

Correspondence should be addressed to Dai Hai Nguyen; nguyendaihai0511@gmail.com

Received 3 January 2017; Revised 7 February 2017; Accepted 9 February 2017; Published 26 February 2017

Academic Editor: Fahima Dilnawaz

Copyright (c) 2017 Thi Lan Nguyen et al. This is an open access article distributed under the Creative Commons Attribution License, which permits unrestricted use, distribution, and reproduction in any medium, provided the original work is properly cited.

The formulation of a potential delivery system based on liposomes (Lips) formulated from soy lecithin (SL) for paclitaxel (PTX) was achieved (PTX-Lips). At first, PTX-Lips were prepared by thin film method using SL and cholesterol and then were characterized for their physiochemical properties (particle size, polydispersity index, zeta potential, and morphology). The results indicated that PTX-Lips were spherical in shape with a dynamic light scattering (DLS) particle size of $131 \pm 30.5 \mathrm{~nm}$. Besides, PTX was efficiently encapsulated in Lips, $94.5 \pm 3.2 \%$ for drug loading efficiency, and slowly released up to $96 \mathrm{~h}$, compared with free PTX. More importantly, cell proliferation kit I (MTT) assay data showed that Lips were biocompatible nanocarriers, and in addition the incorporation of PTX into Lips has been proven successful in reducing the toxicity of PTX. As a result, development of Lips using SL may offer a stable delivery system and promising properties for loading and sustained release of PTX in cancer therapy.

\section{Introduction}

Discovered in 1962, paclitaxel (PTX) is one of the most powerful anticancer drugs for various types of solid tumors, especially for breast cancer and advanced ovarian carcinoma. However, PTX has several disadvantages such as poor water solubility, high toxicity, and low bioavailability, which limit its potential clinical application [1-4]. Among approaches to overcome these drawbacks, drug delivery system is suggested to be a promising candidate owing to the knowledge that nanocarriers can efficiently control the pharmacokinetic characteristics of drugs $[5,6]$. This method can deliver medication within desired therapeutic range to abnormal cells without affecting normal cells while maintaining the systemic levels of drugs [7-13].

Considering the variety of nanocarriers, liposomes (Lips), spherical vesicles consisting of at least one phospholipid bilayer, have been investigated as potential carriers for drug delivery applications due to their high biocompatibility, complete biodegradability, low toxicity, and ability to entrap both water- and lipid-soluble functional compounds and simplify specific drug delivery to tumor site. Furthermore, the stability of the functional components encapsulated in Lips can be increased, therefore maintaining their activities in environments that typically result in rapid degradation. In addition, Lips properties differ considerably with regard to lipid composition, particle size, surface charge, and the method of Lips preparation. The rigidity/fluidity and the charge of the bilayer were strongly influenced by the choice of bilayer components, for instance, saturated or unsaturated phospholipids from natural sources such as egg or soybean phosphatidylcholine $[5,14,15]$. Among these choices, the use of soy lecithin (SL), a naturally occurring phospholipid derived from soybeans, not only provides much more permeable Lips but also facilitates large-scale industrial production because of the reduction of production costs as compared with saturated phospholipids [16]. Several studies have been conducted on the benefits of using SL to obtain desired Lips. 


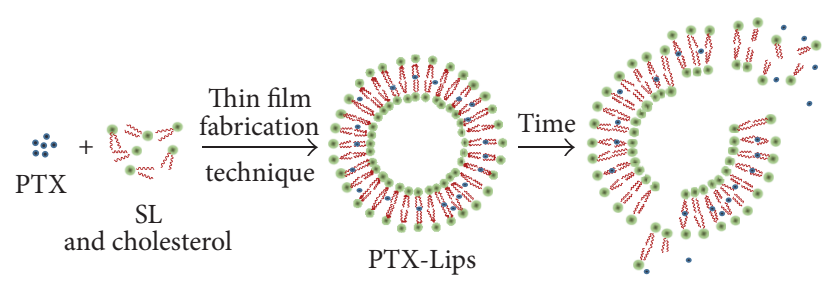

FIGURE 1: Schematic illustration of the formation of PTX-Lips and the release of PTX from Lips over time.

Madrigal-Carballo et al. prepared multilayered biopolymercoated Lips formulated from SL as a novel system for ellagic acid delivery. They successfully achieved monodispered and stable spherical Lips with a diameter of $386.5 \pm 25.9 \mathrm{~nm}$ and a surface charge of $-30.66 \pm 1.55 \mathrm{~nm}$ by thin film fabrication technique for the liposomal system coated with four biopolymer layers. These results indicated that the biopolymer-coated Lips offer good features for loading into their liposomal core and slow release of ellagic acid [17]. Additionally, in a study conducted by Mura and coworkers, Lips made from SL and alkyl polyglucosides (OrNS10) were formulated and characterized for the purpose of designing suitable drug delivery systems for their potential uses. The stability of Lips was also studied by checking average particle size and zeta potential value variation of different liposomal formulations during 4 weeks. The results showed that the addition of OrNS10 to SL has the ability to improve Lips stability [18].

Herein, we developed Lips formulated from SL for PTX delivery (PTX-Lips). The formation of PTX-Lips was prepared according to the thin film method (Figure 1) and later these PTX-Lips were characterized by dynamic light scattering (DLS), zeta potential measurement, and transmission electron microscopy (TEM). Either drug loading or drug release behavior of PTX-Lips was also evaluated. Particularly, cell proliferation kit I (MTT) assay was used to determine the ability of PTX-Lips to minimize the toxicity to HeLa cells of PTX. This study is expected to improve the stability of Lips which was synthesized by eco-friendly SL for PTX delivery in cancer therapy.

\section{Materials and Methods}

2.1. Materials. PTX was supplied by Samyang Corporation (Seoul, Korea). Lecithin from soybean (CAS number 800243-5) and Tween 80 (polyoxyethylene sorbitan monooleate, CAS number 900 5-65-6) were purchased from Tokyo Chemistry Industry Co., Ltd. (Kita-ku, Tokyo, Japan). Cholesterol was obtained from Sigma-Aldrich (St Louis, MO, USA). Cetyltrimethylammonium bromide (CTAB) was purchased from Merck (Darmstadt, Germany). All chemicals and solvents were of highest analytical grade and used without further purification.

\subsection{Methods}

2.2.1. Preparation of PTX-Lips. PTX-Lips were prepared by conventional thin film technique using SL and cholesterol. Briefly, SL (500 mg), cholesterol (56 mg), CTAB (5 mg), and $5 \%$ PTX $(32 \mathrm{mg}$ ) were dissolved in chloroform-methanol $(2: 1 \mathrm{v} / \mathrm{v})$ at room temperature. The mixture was evaporated in a rotary evaporator (Büchi Rotavapor R-114, Essen, Germany) at $45^{\circ} \mathrm{C}$ for $4 \mathrm{~h}$, resulting in a formation of thin lipid film. The obtained thin films were hydrated with $15 \mathrm{~mL}$ of deionized water $\left(\mathrm{deH}_{2} \mathrm{O}\right)$ containing $80 \mathrm{mg}$ of Tween 80 under constant stirring at $60^{\circ} \mathrm{C}$. The suspension was further homogenized (EmulsiFlex-05 homogenizer, Avestin Inc., Ottawa, Canada) at 800 bar for 5 cycles, followed by centrifugation at $5500 \mathrm{rpm}$ for $30 \mathrm{~min}$ to remove nonencapsulated PTX. The resulting sample was then lyophilized using $10 \%$ mannitol as cryoprotectants and stored at $2-8^{\circ} \mathrm{C}$.

2.2.2. Characterization. The particle size and polydispersity index of PTX-Lips were measured by DLS using a Zetasizer Nano ZS (ZEN 3600, Malvern Instruments Ltd., Malvern, Worcestershire, UK). A helium-neon ( $\mathrm{He}-\mathrm{Ne})$ ion laser at $633 \mathrm{~nm}$ was used as the incident beam. The detection angle and the temperature were $90^{\circ}$ and $25^{\circ} \mathrm{C}$, respectively. All samples $(1 \mathrm{mg} / \mathrm{mL}$ ) were sonicated for $15 \mathrm{~min}$, filtered (pore size $=0.45 \mu \mathrm{m}$ ), and carried out at $37^{\circ} \mathrm{C}$. The zeta potential of PTX-Lips was also measured using a Zetasizer Nano ZS ZEN 3600. All measurements were made in triplicate for each sample. The size and morphology of PTX-Lips were confirmed by TEM using JEM-1400 $(300 \mathrm{kV}$; JEOL, Tokyo, Japan). The samples were prepared by placing a drop of solution in $\mathrm{deH}_{2} \mathrm{O}(1 \mathrm{mg} / \mathrm{mL})$ onto a carbon-copper grid (300-mesh, Ted Pella, Inc., USA) and air-drying for $10 \mathrm{~min}$.

2.2.3. PTX Loading Contents and In Vitro PTX Release. In order to determine the PTX loading contents in Lips, PTXLips were first mixed with $1 \%$ Triton X100, incubated for $1 \mathrm{~h}$, and centrifuged at $6000 \mathrm{rpm}$ for $30 \mathrm{~min}$ at $25^{\circ} \mathrm{C}$ to separate PTX from Lips. The total PTX contents in Lips were measured using a Shimadzu LC-20A Prominence System (Shimadzu, Kyoto, Japan). The injected volume was $10 \mu \mathrm{L}$ and the mobile phase (acetonitrile: water $=50: 50 \mathrm{v} / \mathrm{v}$ ) was delivered at $1.00 \mathrm{~mL} / \mathrm{min}$. A reverse-phase Fortis C18 column (150 $4.6 \mathrm{~mm}$ i.d., pore size $5 \mu \mathrm{m}$; Fortis Technologies Ltd., Cheshire, UK) was used, and column effluent was monitored with a UV detector at $227 \mathrm{~nm}$. The calibration curve for quantification of PTX in Lips was found to be linear over the standard PTX concentration range of $0-50,000 \mathrm{ng} / \mathrm{mL}$ with a high correlation coefficient of $R^{2}=0.998$. The following equations were used to calculate the drug loading efficiency (DLE) and drug loading content (DLC):

$$
\begin{aligned}
& \operatorname{DLE}(\%)=\frac{\text { weight of PTX in Lips }}{\text { weight of PTX feed initially }} \times 100, \\
& \operatorname{DLC}(\%)=\frac{\text { weight of PTX inLips }}{\text { weight of Lips and PTX }} \times 100 .
\end{aligned}
$$


The in vitro PTX release experiments were performed in PBS buffer (0.01 M, pH 7.4) at $37^{\circ} \mathrm{C}$ using dialysis method. Initially, $1 \mathrm{~mL}$ of PTX-Lips suspended in PBS containing $2 \%$ Tween 80 was transferred to a dialysis bag (MWCO 6-8 kDa, Spectrum Laboratories, Inc., Rancho Dominguez, CA, USA) and immersed into $20 \mathrm{~mL}$ of the release medium in vials at $37^{\circ} \mathrm{C}$. The vials were then placed in an orbital shaker bath, which was maintained at $37^{\circ} \mathrm{C}$ and shaken horizontally at $100 \mathrm{rpm}$. At specific time intervals, $2 \mathrm{~mL}$ of the release medium was collected and an equal volume of fresh medium was added. The collected samples were filtered (pore size $=0.22 \mu \mathrm{m}$ ) before high performance liquid chromatography analysis.

2.2.4. MTT Viability Test. The MTT assay was used to evaluate cytotoxicity of PTX-Lips on Hela cells. The cells were seeded in a 96-well plate at a density of $1 \times 10^{4}$ cells/well in $130 \mu \mathrm{L}$ of Dulbecco's Modified Eagle's medium (DMEM) supplemented with $10 \%$ FBS and $1 \%$ penicillinstreptomycin and cultured 1 day at $37^{\circ} \mathrm{C}$. Then, the medium was removed and the cells were incubated with samples. The cells were incubated for $48 \mathrm{~h}$, followed by removing medium and washing twice with PBS. MTT solution $(25 \mu \mathrm{L})$ and culture medium $(130 \mu \mathrm{L})$ were added to each well and the cells were cultured for $3 \mathrm{~h}$. DMSO $(130 \mu \mathrm{L})$ was added to each well to dissolve the precipitate. The cells cultured with medium only were used as a control and assigned to $100 \%$ survival. The absorbance was measured at $570 \mathrm{~nm}$ using a multiplate reader (SpectraMax M2e, Molecular Devices Co., USA). Cell viability of all other groups was calculated by normalization of its absorbance intensity to that of "Ctrl" group with the following equation:

$$
\begin{aligned}
\text { Cell viability }(\%)= & \frac{\left([\mathrm{Abs}]_{\text {sample }}-[\mathrm{Abs}]_{\text {blank }}\right)}{\left([\mathrm{Abs}]_{\text {control }}-[\mathrm{Abs}]_{\text {blank }}\right)} \\
& \times 100 \% .
\end{aligned}
$$

\section{Results and Discussion}

3.1. Characterization of PTX-Lips. Two of the most important properties for in vivo integrity and biological fate of nanoparticles (NPs) are particle size and surface charge. In other words, development of carriers with appropriate size and charge plays a crucial role in the field of drug delivery $[19,20]$. Several early studies have reported that the cellular uptake efficiency of NPs decreases when increasing the particle size. It is stated that NPs in the range of 100-200 nm have the highest potential to extend circulation time in the bloodstream because they are small enough to avoid mechanical filtration by the spleen, but large enough to avoid selective uptake in the liver. Small size permits NPs passively targeting tumor cells through the enhanced permeability and retention (EPR) effect, improving intracellular accumulation and localization of NPs in tumor area [21, 22]. Another important parameter that controls the stability of NPs in physiological condition is zeta potential. Not only does negative charge in particular improve the physical stability of Lips by preventing them from fusion and aggregation but also the negatively charged NPs are phagocytized significantly less than positive ones [23]. Therefore, particle size and zeta potential are the two key parameters, which have been proven effective for drug delivery applications.

As shown in Figures 2(b) and 2(d), the DLS particle size of Lips and PTX-Lips and their population standard deviation were $167 \pm 39.1 \mathrm{~nm}$ and $131 \pm 30.5 \mathrm{~nm}$, whereas the polydispersity index values were $0.286 \pm 0.01$ and $0.339 \pm 0.02$, respectively. These results indicated that the particle sizes of Lips and PTX-Lips were not significantly different and their distributions were quite narrow, respectively. Furthermore, the corresponding TEM images (Figures 2(a) and 2(c)) showed that Lips and PTX-Lips were spherical in shape with diameter range of $<200 \mathrm{~nm}$ and without aggregation or fusion, which were correlated with the values of DLS measurement. Besides particle size, zeta potential values of Lips were approximately $-41.7 \mathrm{mV}$ and showed an increase in the surface charge intensity upon inclusion of PTX, $-54.3 \mathrm{mV}$, which may be caused by the PTX interaction with lipid bilayers (Figure 3). Taken together, PTX-Lips might serve as stable spherical nanocarriers with long term circulation in the bloodstream.

3.2. Loading and In Vitro Release of PTX. DLE is an important property in drug-loaded nanocarriers and directly affects the therapeutic effect of the system. The higher the encapsulation capacity NPs have, the larger the number of drugs released at the tumor site $[24,25]$. In this study, the DLE and DLC of PTX-Lips were found to be $94.5 \pm 3.2 \%$ and $4.48 \pm$ $0.47 \%$, respectively. In comparison with other studies, Jiang et al. developed novel dual-functional Lips, PTX-loaded 1,2-distearoyl-sn-glycero-3-phosphoethanolamine- (DSPE-) peptide ${ }_{\mathrm{D}}[\mathrm{KLAKLAK}]_{2}$ (KLA) 2,3-dimethylmaleic anhydride (DMA) Lips (DKD/PTX-Lips), to overcome multidrug resistance. The results showed that the DLE of DKD/PTXLips was $81.8 \pm 0.7 \%$ [26]. In another previous study by Zhou et al., the mitochondrial targeting d-a-tocopheryl polyethylene glycol 1000 succinate- $\left(\mathrm{TPGS}_{1000^{-}}\right)$triphenylphosphine (TPP) conjugate TPGS $_{1000}-\mathrm{TPP}$ ) was synthesized and surface-modified onto PTX-Lips. The targeting PTX-Lips were successfully prepared with DLE of $86.27 \pm 3.15 \%$ [27]. These results demonstrated that the prepared Lips with the high DLE have the potential to be delivered more efficiently to tumor tissues.

In vitro release profiles of free PTX and PTX from PTX-Lips were performed in order to evaluate the stability and release behavior of PTX-Lips. As shown in Figure 4, the prepared Lips showed a long term stable drug release profile up to $96 \mathrm{~h}$. The cumulative release amount of PTX in first $2 \mathrm{~h}$ was around $11 \%$ as compared with $60 \%$ of free PTX. The initial release of PTX could be explained by the PTX molecules, which were absorbed into the outer phospholipid bilayers of Lips. Zhou et al. reported that the initial release of PTX from the targeting PTX-Lips was less than $30 \%$ during the first $2 \mathrm{~h}$ [27]. Moreover, total release amount of PTX was $56 \%$ after $96 \mathrm{~h}$, compared with $97 \%$ of free PTX; in other words, the release behavior of free 


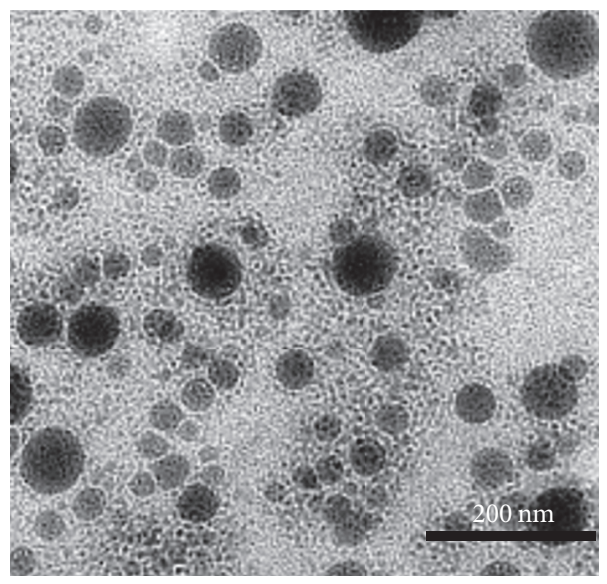

(a)

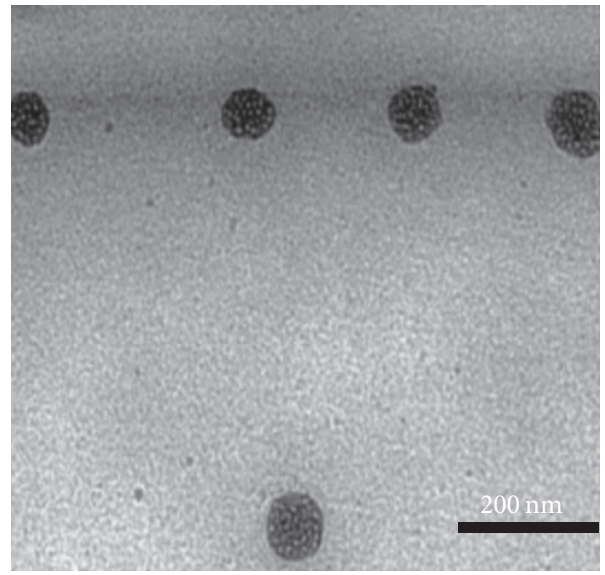

(c)

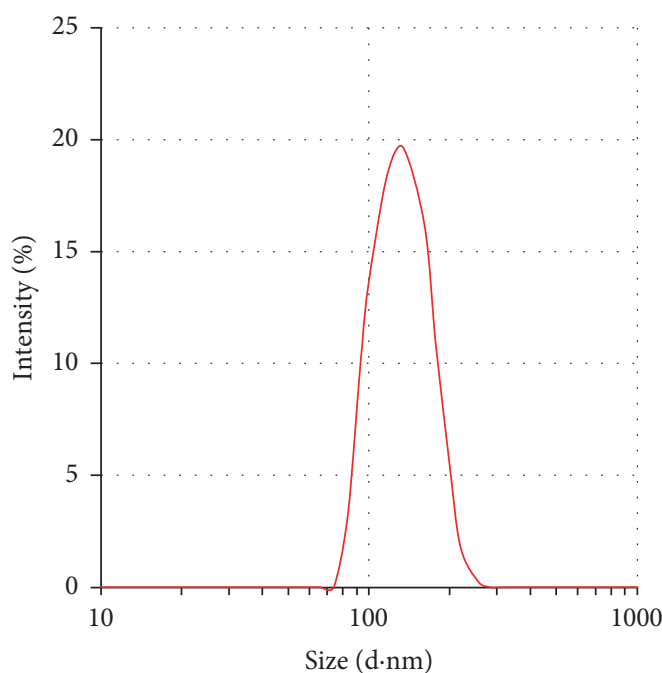

(b)

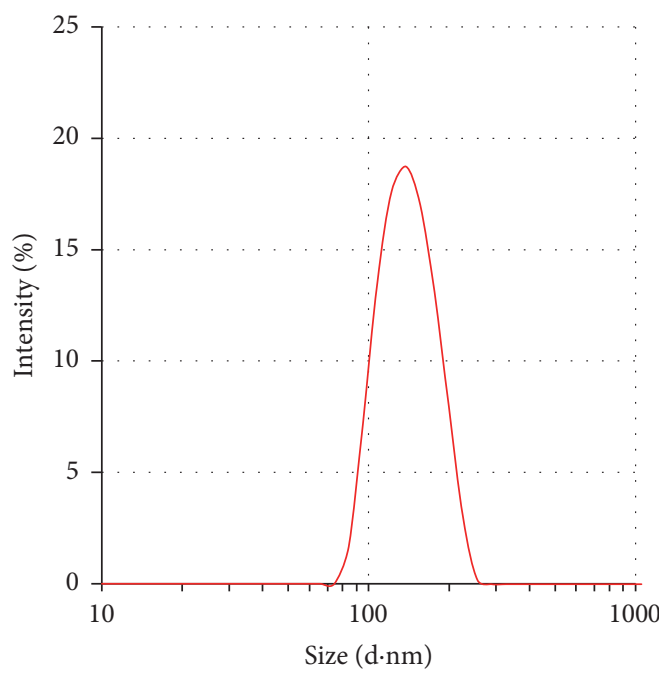

(d)

Figure 2: (a, c) TEM image and (b, d) particle size distribution by DLS of Lips and PTX-Lips, respectively.

PTX was significantly faster than PTX in the prepared Lips. This means that the release rate of PTX was dependent on the presence of Lips. Thus, PTX-Lips may serve as stable NPs, therefore increasing drug accumulation into tumor sites.

3.3. In Vitro Cytotoxicity. Biocompatibility of a material is an important factor for its success in biomedical applications. In this study, MTT assay was carried out to suggest the biocompatibility of PTX-Lips. Figure 5 illustrates the inhibitory effects of free PTX, PTX-Lips, and free PTX of Lips on HeLa cells. The blank Lips showed no obvious cytotoxicity towards HeLa cells. Almost $100 \%$ of cells were still viable at $500 \mu \mathrm{g} / \mathrm{mL}$ of samples for 2 days, indicating that Lips are biocompatible. On the other hand, the cells growth was significantly inhibited when they were treated with PTXLips. A dose-dependent cytotoxicity was observed when individually incubating various doses of free PTX and PTXLips with HeLa cells. The majority of cells were killed when they were treated with PTX at concentration of $10 \mu \mathrm{g} / \mathrm{mL}$ for 2 days. The inhibitory effects of Lips and PTX-Lips were consistent with previous researches. It is expected that the toxicity of PTX would be reduced after being encapsulated in Lips. As shown in Figure 5(b)-(A), the percentage of viable cells of PTX-Lips at equivalent PTX concentration of $10 \mu \mathrm{g} / \mathrm{mL}$ was around $64 \%$ as compared with that only around $12 \%$ of free PTX. These results clearly confirmed that PTXLips have the great inhibitory effect against HeLa cells and could be safely used as drug delivery vehicles for in vivo applications. 


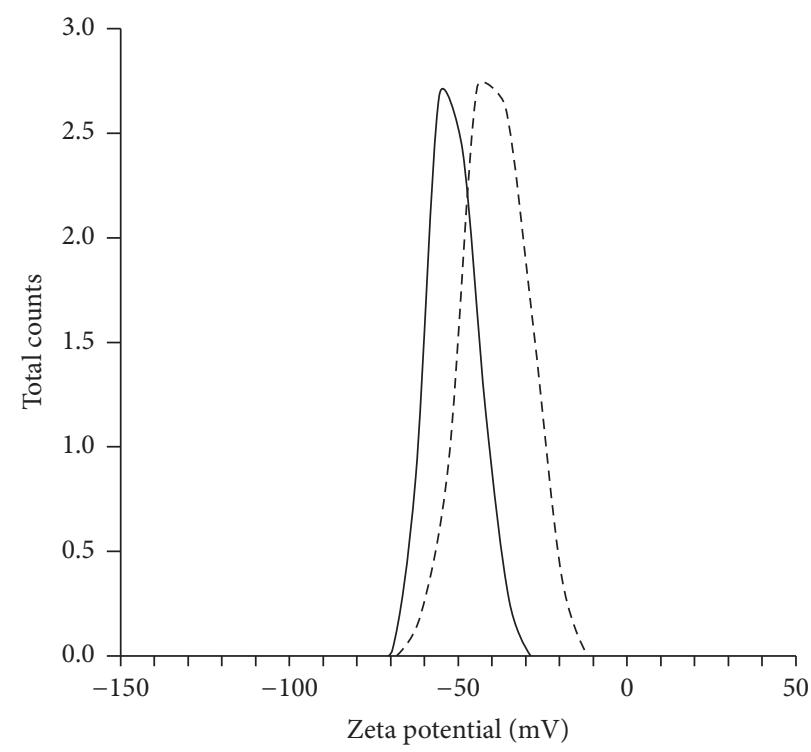

FIGURE 3: Nanoparticle surface charge via zeta potential of Lips (dashed line) and PTX-Lips (solid line).

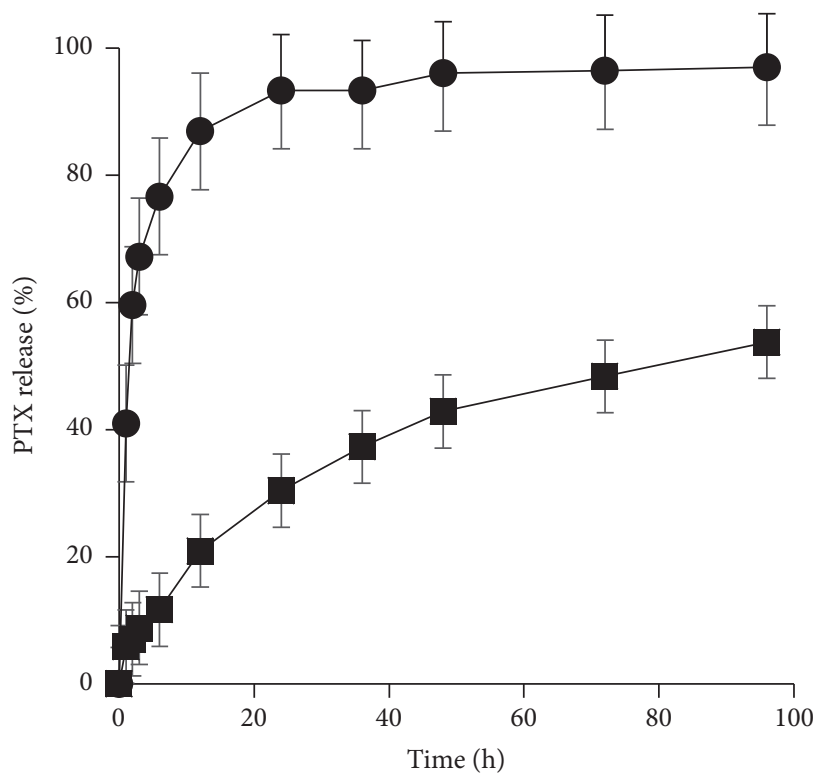

FIgURE 4: In vitro release profiles of free PTX (circle) and PTX from PTX-Lips (square).

\section{Conclusion}

Liposomal delivery systems for PTX have been successfully developed by thin film technique. The prepared PTX-Lips were spherical in shape with a diameter around $131 \mathrm{~nm}$, which would be suitable for in vivo drug release. The NPs had DLE and DLC of $94.5 \pm 3.2 \%$ and $4.48 \pm 0.47 \%$ and in addition, the release profile showed sustained release of PTX, respectively. Particularly, it was clear that PTX-Lips could reduce the toxicity of PTX determined by MTT assay. Our results suggest that the Lips made from natural SL have the potential as stable, biocompatible, and efficient PTX delivery systems for the treatment of cancer.

\section{Competing Interests}

The authors declare that there is no conflict of interests regarding the publication of this paper. 
(A)
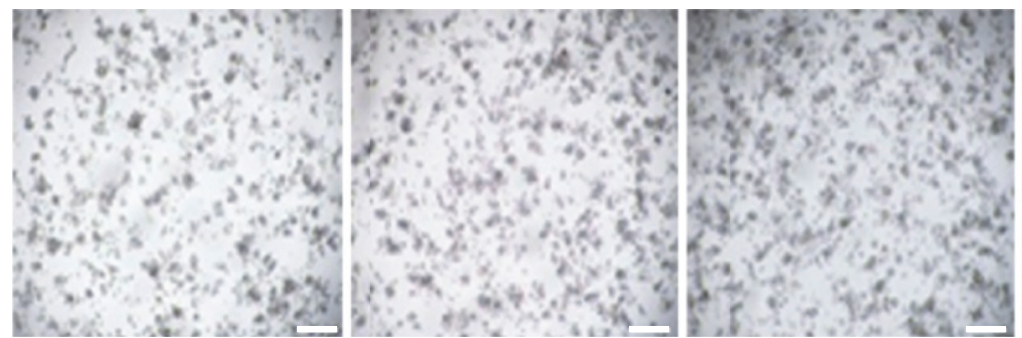

$500 \mu \mathrm{g}$

$250 \mu \mathrm{g}$

$100 \mu \mathrm{g}$

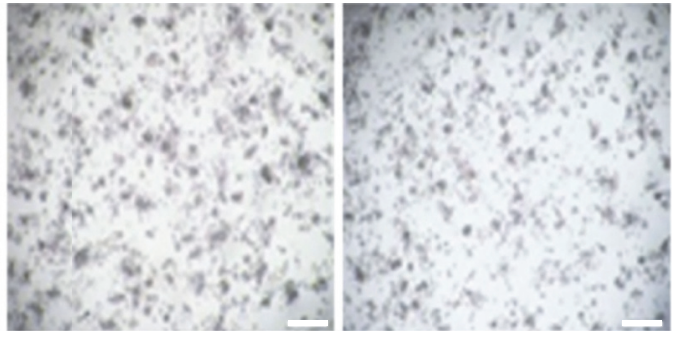

$50 \mu \mathrm{g}$

$0 \mu \mathrm{g}$

(B)

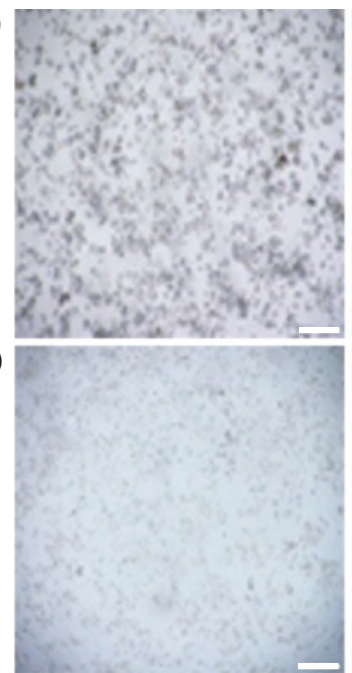

$20 \mu \mathrm{g}$

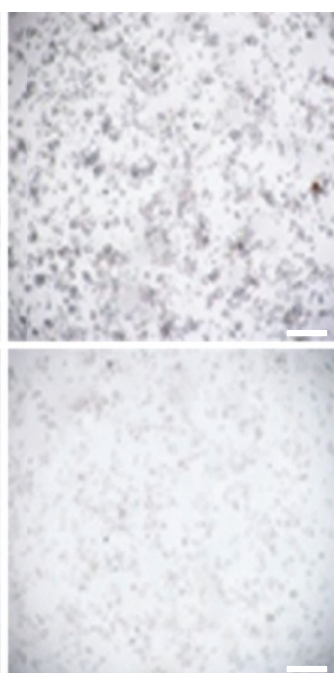

$10 \mu \mathrm{g}$

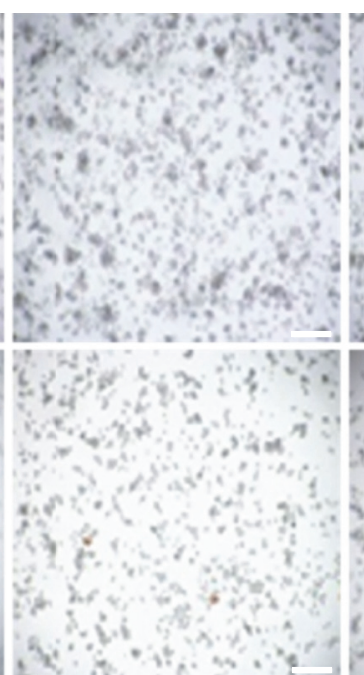

$5 \mu \mathrm{g}$
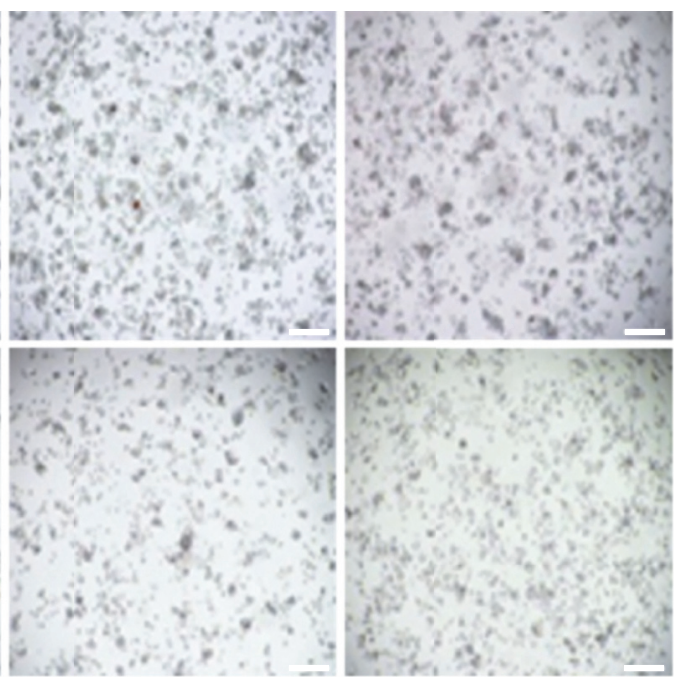

$1 \mu \mathrm{g}$

$0.1 \mu \mathrm{g}$

(a)
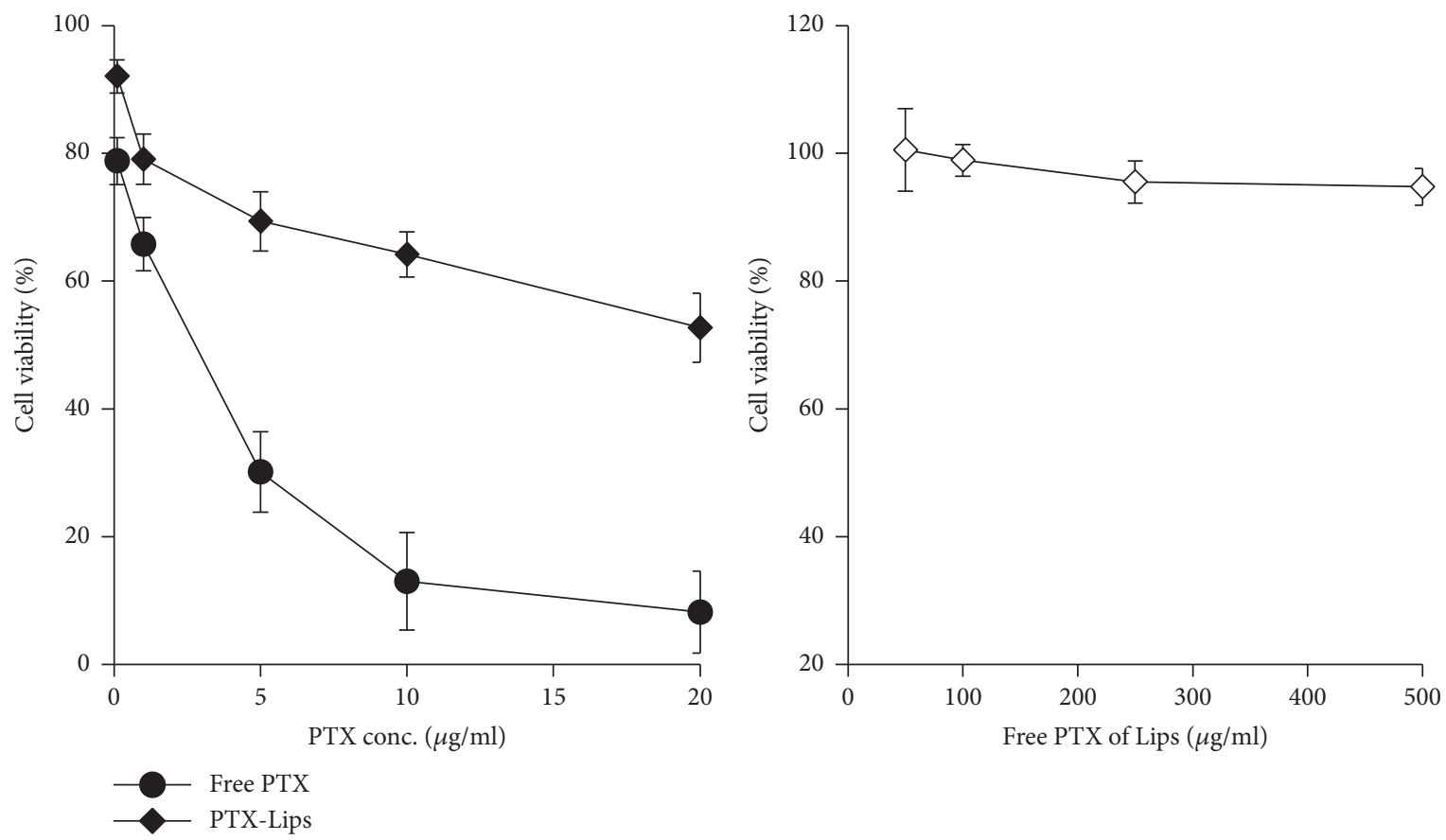

(A)

(B)

(b)

FIGURE 5: (a) Images of HeLa cells incubated with (A) Lips at different concentrations, (B) PTX-Lips, and (C) free PTX at different PTX doses observed under microscope for $48 \mathrm{~h}($ scale bar $=80 \mu \mathrm{m}$ ) and (b) viability of HeLa cells incubated with (A) free PTX, PTX-Lips at different PTX doses, and (B) free PTX of Lips at different concentrations for $48 \mathrm{~h}$. The cells were exposed to the samples for the indicated times. The data represent the mean values \pm the standard deviation $(\mathrm{SD})(n=4)$. 


\section{Acknowledgments}

This research was funded by the Development of Science and Technology (DOST) under Grant no. 69/2016, date: 18 July 2016.

\section{References}

[1] T. Zhao, H. Chen, Y. Dong et al., "Paclitaxel-loaded poly(Glycolide-co- $\varepsilon$ caprolactone)-b-d- $\alpha$-tocopheryl polyethylene glycol 2000 succinate nanoparticles for lung cancer therapy," International Journal of Nanomedicine, vol. 8, pp. 1947-1957, 2013.

[2] R. M. Taylor and L. O. Sillerud, "Paclitaxel-loaded iron platinum stealth immunomicelles are potent MRI imaging agents that prevent prostate cancer growth in a PSMA-dependent manner," International Journal of Nanomedicine, vol. 7, pp. 4341-4352, 2012.

[3] T. Konno, J. Watanabe, and K. Ishihara, "Enhanced solubility of paclitaxel using water-soluble and biocompatible 2methacryloyloxyethyl phosphorylcholine polymers," Journal of Biomedical Materials Research, vol. 65, no. 2, pp. 209-214, 2003.

[4] D. Q. Hoang, T. V. Tran, N. Q. Tran et al., "Functionalization of $\mathrm{Fe}_{3} \mathrm{O}_{4}$ nanoparticles with biodegradable chitosan-graftedmPEG for paclitaxel delivery," Green Processing and Synthesis, vol. 5, no. 5, pp. 459-466, 2016.

[5] D. H. Nguyen, J. S. Lee, J. H. Choi et al., "Heparin nanogelcontaining liposomes for intracellular RNase delivery," Macromolecular Research, vol. 23, no. 8, pp. 765-769, 2015.

[6] H. D. Nguyen, T. D. Nguyen, D. H. Nguyen, and P. T. Nguyen, "Magnetic properties of $\mathrm{Cr}$ doped $\mathrm{Fe}_{3} \mathrm{O}_{4}$ porous nanoparticles prepared through a co-precipitation method using surfactant," Advances in Natural Sciences: Nanoscience and Nanotechnology, vol. 5, no. 3, 2014.

[7] M. Goldberg, R. Langer, and X. Jia, "Nanostructured materials for applications in drug delivery and tissue engineering," Journal of Biomaterials Science, Polymer Edition, vol. 18, no. 3, pp. 241-268, 2007.

[8] D. H. Nguyen, J. S. Lee, J. H. Choi, K. M. Park, Y. Lee, and K. D. Park, "Hierarchical self-assembly of magnetic nanoclusters for theranostics: tunable size, enhanced magnetic resonance imagability, and controlled and targeted drug delivery," Acta Biomaterialia, vol. 35, pp. 109-117, 2016.

[9] T. T. Nguyen Thi, T. V. Tran, N. Q. Tran, C. K. Nguyen, and D. H. Nguyen, "Hierarchical self-assembly of heparin-PEG end-capped porous silica as a redox sensitive nanocarrier for doxorubicin delivery," Materials Science and Engineering C, vol. 70, part 2, pp. 947-954, 2017.

[10] D. H. Nguyen, J. Hoon Choi, Y. Ki Joung, and K. Dong Park, "Disulfide-crosslinked heparin-pluronic nanogels as a redoxsensitive nanocarrier for intracellular protein delivery," Journal of Bioactive and Compatible Polymers, vol. 26, no. 3, pp. 287-300, 2011.

[11] D. H. Nguyen, Y. K. Joung, J. H. Choi, H. T. Moon, and K. D. Park, "Targeting ligand-functionalized and redox-sensitive heparin-Pluronic nanogels for intracellular protein delivery," Biomedical Materials, vol. 6, no. 5, Article ID 055004, 2011.

[12] D. H. Nguyen, J. W. Bae, J. H. Choi, J. S. Lee, and K. D. Park, "Bioreducible cross-linked Pluronic micelles: PHtriggered release of doxorubicin and folate-mediated cellular uptake," Journal of Bioactive and Compatible Polymers, vol. 28, no. 4, pp. 341-354, 2013.
[13] T. D. van, N. Q. Tran, D. H. Nguyen, C. K. Nguyen, D. L. Tran, and P. T. Nguyen, "Injectable hydrogel composite based gelatinPEG and biphasic calcium phosphate nanoparticles for bone regeneration," Journal of Electronic Materials, vol. 45, no. 5, pp. 2415-2422, 2016.

[14] A. Akbarzadeh, R. Rezaei-Sadabady, S. Davaran et al., "Liposome: classification, preparation, and applications," Nanoscale Research Letters, vol. 8, article no. 102, 2013.

[15] S. K. Sahoo and V. Labhasetwar, "Nanotech approaches to drug delivery and imaging," Drug Discovery Today, vol. 8, no. 24, pp. 1112-1120, 2003.

[16] P. P. Thi and D. H. Nguyen, "Gelatin as an ecofriendly natural polymer for preparing colloidal silver@gold nanobranches," Green Processing and Synthesis, vol. 5, no. 5, pp. 467-472, 2016.

[17] S. Madrigal-Carballo, S. Lim, G. Rodriguez et al., "Biopolymer coating of soybean lecithin liposomes via layer-by-layer selfassembly as novel delivery system for ellagic acid," Journal of Functional Foods, vol. 2, no. 2, pp. 99-106, 2010.

[18] S. Mura, M. Manconi, S. Madrigal-Carballo et al., "Composite soy lecithin-decylpolyglucoside vesicles: a theoretical and experimental study," Colloids and Surfaces A: Physicochemical and Engineering Aspects, vol. 323, no. 1-3, pp. 175-179, 2008.

[19] Y. Yoshizawa, Y. Kono, K.-I. Ogawara, T. Kimura, and K. Higaki, "PEG liposomalization of paclitaxel improved its in vivo disposition and anti-tumor efficacy," International Journal of Pharmaceutics, vol. 412, no. 1-2, pp. 132-141, 2011.

[20] P. P. N. Thi, M. T. Nguyen, and D. H. Nguyen, "Role of collagen concentration in stability of Star-shaped Silver@Gold nanoparticles," Journal of Nano Research, vol. 40, pp. 113-119, 2016.

[21] B. Aslan, B. Ozpolat, A. K. Sood, and G. Lopez-Berestein, "Nanotechnology in cancer therapy," Journal of Drug Targeting, vol. 21, no. 10, pp. 904-913, 2013.

[22] D. H. Nguyen, J. S. Lee, J. W. Bae et al., "Targeted doxorubicin nanotherapy strongly suppressing growth of multidrug resistant tumor in mice," International Journal of Pharmaceutics, vol. 495, no. 1, pp. 329-335, 2015.

[23] Y. Sheng, L. Chang, T. Kuang, and J. Hu, "PEG/heparindecorated lipid-polymer hybrid nanoparticles for longcirculating drug delivery," RSC Advances, vol. 6, no. 28, pp. 23279-23287, 2016.

[24] H. Li, J. Z. Zhang, Q. Tang, M. Du, J. Hu, and D. Yang, "Reduction-responsive drug delivery based on mesoporous silica nanoparticle core with crosslinked poly(acrylic acid) shell," Materials Science and Engineering C, vol. 33, no. 6, pp. 3426-3431, 2013.

[25] T. U. Ly, N. Q. Tran, T. K. D. Hoang, K. N. Phan, H. N. Truong, and C. K. Nguyen, "Pegylated dendrimer and its effect in fluorouracil loading and release for enhancing antitumor activity," Journal of Biomedical Nanotechnology, vol. 9, no. 2, pp. 213-220, 2013.

[26] L. Jiang, L. Li, X. He et al., "Overcoming drug-resistant lung cancer by paclitaxel loaded dual-functional liposomes with mitochondria targeting and $\mathrm{pH}$-response," Biomaterials, vol. 52, no. 1, pp. 126-139, 2015.

[27] J. Zhou, W.-Y. Zhao, X. Ma et al., "The anticancer efficacy of paclitaxel liposomes modified with mitochondrial targeting conjugate in resistant lung cancer," Biomaterials, vol. 34, no. 14, pp. 3626-3638, 2013. 

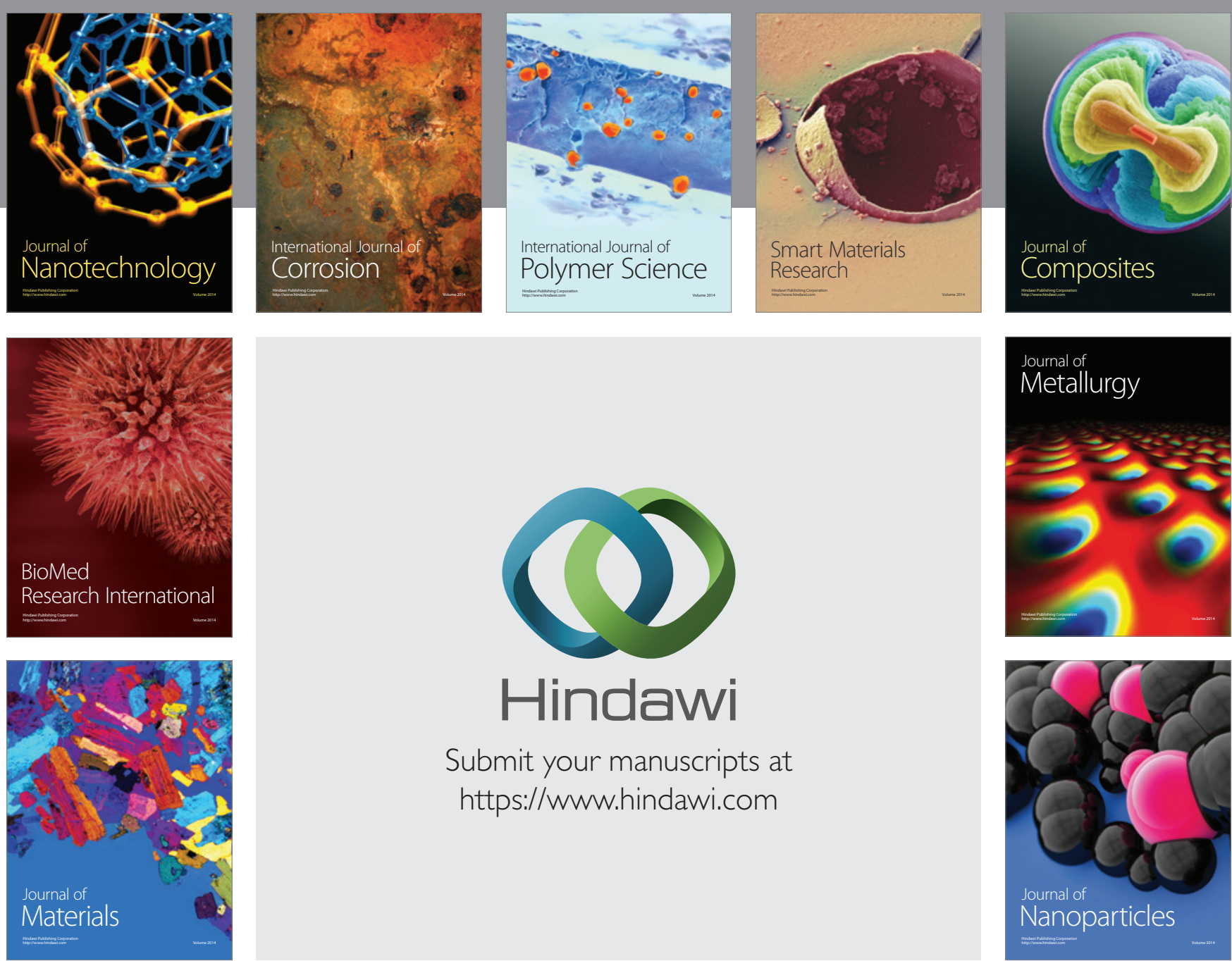

\section{Hindawi}

Submit your manuscripts at

https://www.hindawi.com

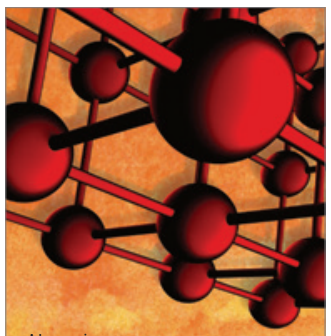

Materials Science and Engineering
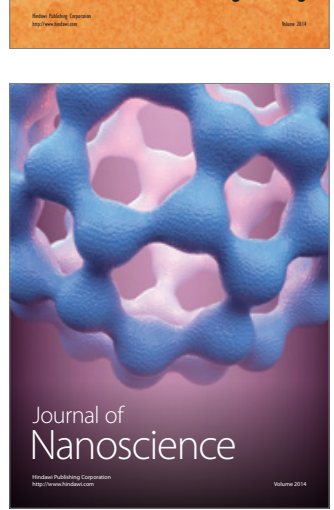
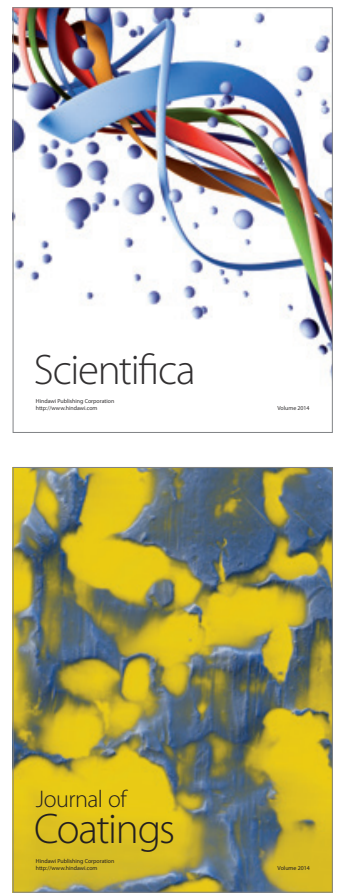
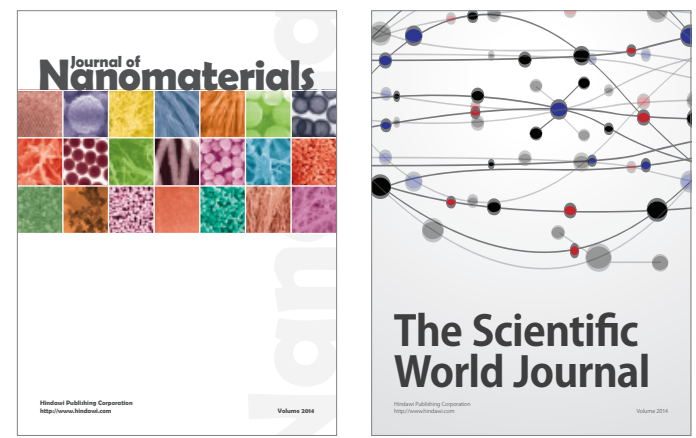

The Scientific World Journal
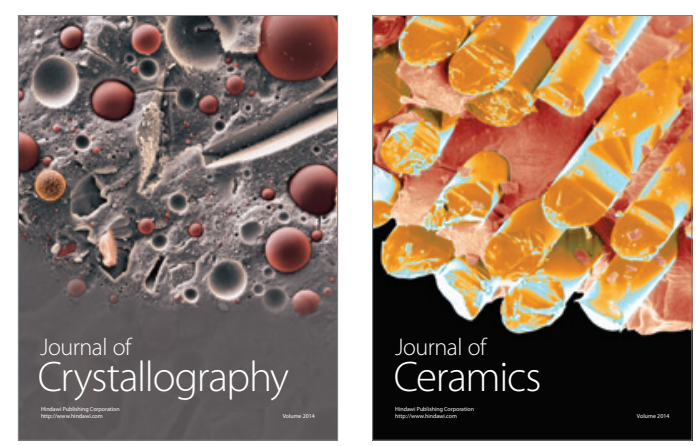
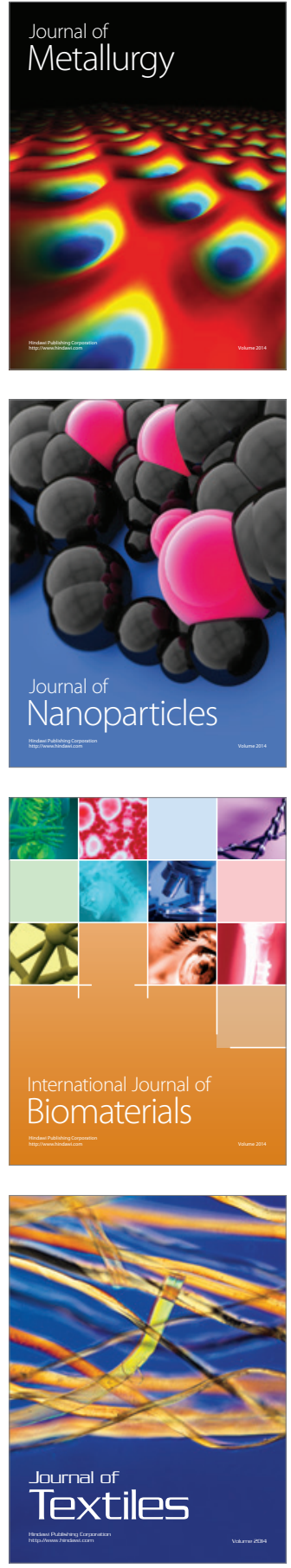